\title{
Higher-order boundary value problems for Carathéodory differential inclusions
}

\author{
M. Aitalioubrahim and S. Sajid
}




\title{
HIGHER-ORDER BOUNDARY VALUE PROBLEMS FOR CARATHÉODORY DIFFERENTIAL INCLUSIONS
}

\author{
M. AITALIOUBRAHIM AND S. SAJID
}

Received 10 September, 2007

Abstract. In this paper we prove existence results for boundary value problems for higher-order differential inclusion $x^{(n)}(t) \in F(t, x(t))$ with nonlocal boundary conditions, where $F$ is a compact convex $L^{1}$-Carathéodory multifunction.

2000 Mathematics Subject Classification: 34A60, 34B10, 34B15

Keywords: boundary value problems, Carathéodory multifunction, fixed point

\section{INTRODUCTION}

The aim of this paper is to establish the existence of solutions of the higher-order boundary value problems

$$
\left\{\begin{array}{l}
x^{(n)}(t) \in F(t, x(t)) \quad \text { a.e. on }[0,1] \\
x^{(i)}(0)=0, \quad 0 \leq i \leq n-2 \\
x(\eta)=x(1)
\end{array}\right.
$$

with $n \geq 2$,

$$
\left\{\begin{array}{l}
x^{(n)}(t) \in F(t, x(t)) \quad \text { a.e. on }[0,1] \\
x(0)=x^{\prime}(\eta) \\
x(1)=x(\tau)
\end{array}\right.
$$

with $n \geq 2$,

with $n \geq 4$, and

$$
\left\{\begin{array}{l}
x^{(n)}(t) \in F(t, x(t)) \quad \text { a.e. on }[0,1], \\
x^{(i)}(0)=x^{(i+1)}(\eta), \quad 2 \leq i \leq n-2, \\
x(0)=x^{\prime}(\eta) \\
x(1)=x(\tau)
\end{array}\right.
$$

$$
\left\{\begin{array}{l}
x^{(n)}(t) \in F(t, x(t)) \quad \text { a.e. on }[0,1], \\
x^{(i)}(0)=x^{(i+1)}(\eta), \quad 0 \leq i \leq n-2
\end{array}\right.
$$


with $n \geq 2$, where $F:[0,1] \times \mathbb{R} \rightarrow 2^{\mathbb{R}}$ is a compact convex $L^{1}$-Carathéodory multivalued map and $(\eta, \tau) \in] 0,1[2$.

Three and four-point boundary value problems for second-order differential inclusions was initiated by Benchohra and Ntouyas [1,2]. The authors proved the existence of solutions on compact intervals for the problems (1.1) and (1.2) in the particular case $n=2$. In order to obtain solutions of (1.1) and (1.2), the authors reduce the existence of solutions to the search for fixed points of a suitable multi-valued map on the Banach space $C([0,1], \mathbb{R})$. Indeed, they used the fixed point theorem for condensing maps due to Martelli [5].

In this paper, we extend the results of Benchohra and Ntouyas [1,2] to the $n$th order boundary value problems and prove the existence of solutions of (1.3) and (1.4). We shall adopt the techniques used by Benchohra and Ntouyas in the previous papers.

\section{Preliminaries}

Let $(E,\|\cdot\|)$ be a Banach space. We denote by $C([0,1], E)$ the Banach space of continuous functions from $[0,1]$ to $E$ equipped with the norm $\|x\|_{\infty}:=\sup \{\|x(t)\|:$ $t \in[0,1]\}$. A multifunction is said to be measurable if its graph is measurable. For more details on measurability theory, we refer the reader to the book of Castaing and Valadier [3].

Definition 1. A multi-valued map $F:[0,1] \times \mathbb{R} \rightarrow 2^{\mathbb{R}}$ is said to be an $L^{1}$-Carathéodory if

(i) $t \mapsto F(t, x)$ is measurable for all $x \in \mathbb{R}$;

(ii) $x \mapsto F(t, x)$ is upper semi-continuous for almost all $t \in[0,1]$;

(iii) For each $k>0$, there exists $h_{k} \in L^{1}\left([0,1] ; \mathbb{R}^{+}\right)$such that

$$
\|F(t, x)\|:=\sup \{\|y\|: y \in F(t, x)\} \leq h_{k}(t)
$$

for all $\|x\| \leq k$ and for almost all $t \in[0,1]$.

Definition 2. Let $E$ be a separable Banach space, $X$ a nonempty subset of $E$, and $G: X \rightarrow 2^{E}$ a multi-valued map. We say that:

(1) $G$ is upper semi-continuous on $X$ if for each $x \in X$ the set $G(x)$ is a nonempty closed subset of $E$ and if, for each open set $B$ of $E$ containing $G(x)$, there exists an open neighborhood $V$ of $x$ such that $G(V) \subset B$.

(2) $G$ has a fixed point if there is $x \in X$ such that $x \in G(x)$.

(3) $G$ is said to be completely continuous if $G(B)$ is relatively compact for every $B$ bounded set of $X$.

(4) If $G$ is upper semi-continuous, it is said to be condensing map if, for any subset $B \subset X$ with $\alpha(B) \neq 0$, we have

$$
\alpha(G(B))<\alpha(B),
$$

where $\alpha$ denotes the Kuratowski measure of noncompactness.*

*Note that a completely continuous multivalued map is the easiest example of a condensing map. 
It is known that if the multi-valued map $G$ is completely continuous with nonempty compact values, the $G$ is upper semi-continuous if and only if $G$ has a closed graph.

Definition 3. A function $x:[0,1] \rightarrow \mathbb{R}$ is said to be solution of (1.1) (resp., (1.2), (1.3), (1.4)) if $x$ is $(n-1)$-times differentiable, $x^{(n-1)}$ is absolutely continuous and $x$ satisfies the conditions of (1.1) (resp., (1.2), (1.3), (1.4)).

Let $\eta \in \mathbb{R}$ and $n \in \mathbb{N} \backslash\{0,1\}$. For the techniques reasoning, we will need, in the sequel, the sequence of functions $\left(\varphi_{p}\right)_{2 \leq p \leq n}$ defined by the following formulas.

For all $t \in[0,1]$, we put

$$
\begin{aligned}
& \varphi_{2}(t)=1 \\
& \varphi_{3}(t)=t+\varphi_{2}(\eta) \\
& \varphi_{p}(t)=\frac{t^{p-2}}{(p-2) !}+\sum_{k=3}^{p-1} \varphi_{k-1}(\eta) \frac{t^{p-k}}{(p-k) !}+\varphi_{p-1}(\eta) .
\end{aligned}
$$

Remark 1. The following assertions hold:

(a) For all $t \in[0,1]$ and $k \in\{0, \ldots, n-2\}, \varphi_{n}^{(k)}(t)=\varphi_{n-k}(t)$;

(b) For all $k \in\{0, \ldots, n-3\}, \varphi_{n-k}(0)=\varphi_{n-k-1}(\eta)$;

(c) For all $k \in\{0, \ldots, n-2\}$, the function $\varphi_{n}^{(k)}$ is increasing.

\section{MAIN RESULTS}

Assume that the following hypotheses hold:

$\left(H_{1}\right) F:[0,1] \times \mathbb{R} \rightarrow 2^{\mathbb{R}}$ is an $L^{1}$-Carathéodory set-valued map with nonempty compact convex values;

$\left(H_{2}\right)$ There exists a function $m \in L^{1}\left([0,1], \mathbb{R}^{+}\right)$such that

$$
\|F(t, x)\| \leq m(t)
$$

for almost all $t \in[0,1]$ and all $x \in \mathbb{R}$.

We shall prove the following main results.

Theorem 1. If assumptions $\left(H_{1}\right)$ and $\left(H_{2}\right)$ are satisfied, then problem (1.1) has at least one solution on $[0,1]$.

Theorem 2. If assumptions $\left(H_{1}\right)$ and $\left(H_{2}\right)$ are satisfied, then problems (1.2) and (1.3) have at least one solution on $[0,1]$.

Theorem 3. If assumptions $\left(H_{1}\right)$ and $\left(H_{2}\right)$ are satisfied, then problem (1.4) has at least one solution on $[0,1]$. 


\section{Proof of The Main Results}

For $y \in C([0,1], \mathbb{R})$, we set

$$
S_{F}(y):=\left\{g \in L^{1}([0,1], \mathbb{R}): g(t) \in F(t, y(t)) \text { for a. e. } t \in[0,1]\right\} .
$$

In the sequel, we will use the following important lemmas. They will play a crucial role in the proof of the main results.

Lemma 1 ([4]). If $\operatorname{dim}(E)<\infty$ and $F:[0,1] \times E \rightarrow 2^{E}$ is compact and convex then $S_{F}(y) \neq \varnothing$ for all $y \in E$.

Lemma 2 ([4]). If $F$ satisfies $\left(H_{1}\right)$ and $S_{F} \neq \varnothing$ then, for any linear continuous mapping $\Gamma: L^{1}([0,1], E) \rightarrow C([0,1], E)$, the convex compact multi-function $\Gamma \circ S_{F}: C([0,1], E) \rightarrow 2^{C([0,1], E)}$ has a closed graph.

Lemma 3 ([5]). Let $T: E \rightarrow 2^{E}$ be a convex compact condensing multi-valued mapping. If the set

$$
\Omega:=\{y \in E: \lambda y \in T(y) \text { for some } \lambda>1\}
$$

is bounded, then $T$ has a fixed point.

Proof of Theorem 1. By Lemma 1 , for $y \in C([0,1], \mathbb{R}), S_{F}(y)$ is nonempty. Let us transform the problem into a fixed point problem. Consider the multi-valued map $T: C([0,1], \mathbb{R}) \rightarrow 2^{C([0,1], \mathbb{R})}$ defined as follows: for $y \in C([0,1], \mathbb{R}), T(y)$ is the set of all $z \in C([0,1], \mathbb{R})$ such that

$$
\begin{aligned}
z(t)=\int_{0}^{t} \frac{(t-s)^{n-1}}{(n-1) !} g(s) d s+\frac{t^{n-1}}{1-\eta^{n-1}} \int_{0}^{\eta} & \frac{(\eta-s)^{n-1}}{(n-1) !} g(s) d s \\
& -\frac{t^{n-1}}{1-\eta^{n-1}} \int_{0}^{1} \frac{(1-s)^{n-1}}{(n-1) !} g(s) d s,
\end{aligned}
$$

where $g \in S_{F}(y)$. We shall show that $T$ satisfies the assumptions of Lemma 3. The proof will be given in several steps:

STEP 1: $T(y)$ is convex for each $y \in C([0,1], \mathbb{R})$. Let $h_{1}, h_{2} \in T(y)$, then

$$
\begin{aligned}
h_{i}(t)=\int_{0}^{t} \frac{(t-s)^{n-1}}{(n-1) !} g_{i}(s) d s+\frac{t^{n-1}}{1-\eta^{n-1}} & \int_{0}^{\eta} \frac{(\eta-s)^{n-1}}{(n-1) !} g_{i}(s) d s \\
& -\frac{t^{n-1}}{1-\eta^{n-1}} \int_{0}^{1} \frac{(1-s)^{n-1}}{(n-1) !} g_{i}(s) d s,
\end{aligned}
$$

where $g_{i} \in S_{F}(y)$ and $i=1,2$. Let $0 \leq \alpha \leq 1$. For all $t \in[0,1]$ we have

$$
\begin{aligned}
\left(\alpha h_{1}+(1-\alpha) h_{2}\right)(t)= & \int_{0}^{t} \frac{(t-s)^{n-1}}{(n-1) !}\left(\alpha g_{1}(s)+(1-\alpha) g_{2}(s)\right) d s \\
& +\frac{t^{n-1}}{1-\eta^{n-1}} \int_{0}^{\eta} \frac{(\eta-s)^{n-1}}{(n-1) !}\left(\alpha g_{1}(s)+(1-\alpha) g_{2}(s)\right) d s
\end{aligned}
$$




$$
-\frac{t^{n-1}}{1-\eta^{n-1}} \int_{0}^{1} \frac{(1-s)^{n-1}}{(n-1) !}\left(\alpha g_{1}(s)+(1-\alpha) g_{2}(s)\right) d s .
$$

The set $S_{F}(y)$ is convex because $F$ is convex. Hence $\alpha h_{1}+(1-\alpha) h_{2} \in T(y)$.

STEP 2: $T$ is bounded on bounded sets of $C([0,1], \mathbb{R})$. Indeed, it is sufficient to show that $T\left(B_{r}\right)$ is bounded for all $r \geq 0$, where $B_{r}=\left\{y \in C([0,1], \mathbb{R}):\|y\|_{\infty} \leq r\right\}$. Let $h \in T\left(B_{r}\right)$. For all $t \in[0,1]$ we have

$$
\begin{aligned}
h(t)=\int_{0}^{t} \frac{(t-s)^{n-1}}{(n-1) !} g(s) d s+\frac{t^{n-1}}{1-\eta^{n-1}} \int_{0}^{\eta} & \frac{(\eta-s)^{n-1}}{(n-1) !} g(s) d s \\
& -\frac{t^{n-1}}{1-\eta^{n-1}} \int_{0}^{1} \frac{(1-s)^{n-1}}{(n-1) !} g(s) d s,
\end{aligned}
$$

where $y \in B_{r}$ and $g \in S_{F}(y)$. Thus, by $\left(H_{2}\right)$,

$$
\begin{aligned}
|h(t)| \leq \int_{0}^{t} \frac{(t-s)^{n-1}}{(n-1) !} m(s) d s+\frac{t^{n-1}}{1-\eta^{n-1}} & \int_{0}^{\eta} \frac{(\eta-s)^{n-1}}{(n-1) !} m(s) d s \\
& +\frac{t^{n-1}}{1-\eta^{n-1}} \int_{0}^{1} \frac{(1-s)^{n-1}}{(n-1) !} m(s) d s .
\end{aligned}
$$

Then

$$
\begin{aligned}
\|h\|_{\infty} \leq \int_{0}^{1} \frac{(1-s)^{n-1}}{(n-1) !} m(s) d s+\frac{1}{1-\eta^{n-1}} & \int_{0}^{\eta} \frac{(\eta-s)^{n-1}}{(n-1) !} m(s) d s \\
& +\frac{1}{1-\eta^{n-1}} \int_{0}^{1} \frac{(1-s)^{n-1}}{(n-1) !} m(s) d s .
\end{aligned}
$$

Hence $T\left(B_{r}\right) \subset B_{\delta}$, where $\delta$ is the right-hand term in the above inequality.

STEP 3: $T$ sends bounded sets of $C([0,1], \mathbb{R})$ into equicontinuous sets. Indeed, let $h \in T\left(B_{r}\right)$. For all $t \in[0,1]$, we have

$$
\begin{aligned}
h(t)=\int_{0}^{t} \frac{(t-s)^{n-1}}{(n-1) !} g(s) d s+\frac{t^{n-1}}{1-\eta^{n-1}} \int_{0}^{\eta} & \frac{(\eta-s)^{n-1}}{(n-1) !} g(s) d s \\
& -\frac{t^{n-1}}{1-\eta^{n-1}} \int_{0}^{1} \frac{(1-s)^{n-1}}{(n-1) !} g(s) d s,
\end{aligned}
$$

where $y \in B_{r}$ and $g \in S_{F}(y)$. Let $t, s \in[0,1]$ be such that $t<s$. We have

$$
\begin{aligned}
\mid h(s)- & h(t) \mid \\
\leq & \int_{t}^{s} \frac{(s-\tau)^{n-1}}{(n-1) !}|g(\tau)| d \tau+\int_{0}^{t} \frac{(s-\tau)^{n-1}-(t-\tau)^{n-1}}{(n-1) !}|g(\tau)| d \tau \\
& +\frac{s^{n-1}-t^{n-1}}{1-\eta^{n-1}}\left(\int_{0}^{\eta} \frac{(\eta-\tau)^{n-1}}{(n-1) !}|g(\tau)| d \tau+\int_{0}^{1} \frac{(1-\tau)^{n-1}}{(n-1) !}|g(\tau)| d \tau\right)
\end{aligned}
$$




$$
\begin{aligned}
\leq & \int_{t}^{s} \frac{(1-\tau)^{n-1}}{(n-1) !} m(\tau) d \tau+\int_{0}^{1} \frac{(s-\tau)^{n-1}-(t-\tau)^{n-1}}{(n-1) !} m(\tau) d \tau \\
& +\frac{s^{n-1}-t^{n-1}}{1-\eta^{n-1}}\left(\int_{0}^{\eta} \frac{(\eta-\tau)^{n-1}}{(n-1) !} m(\tau) d \tau+\int_{0}^{1} \frac{(1-\tau)^{n-1}}{(n-1) !} m(\tau) d \tau\right) .
\end{aligned}
$$

The right-hand side of the above inequality converges to 0 as $s$ tends to $t$. Now, by Steps 1, 2, and 3 combined with the Arzelà-Ascoli theorem, we conclude that $T$ is completely continuous.

STEP 4: $T$ has a closed graph. Let $\left(y_{p}\right)_{p}$ a sequence converging to $y$ and consider a sequence $\left(h_{p}\right)_{p}$ such that $h_{p} \in T\left(y_{p}\right)$ and $\left(h_{p}\right)_{p}$ converges to $h$. We shall prove that $h \in T(y)$. We have

$$
\begin{aligned}
h_{p}(t)=\int_{0}^{t} \frac{(t-s)^{n-1}}{(n-1) !} g_{p}(s) d s+\frac{t^{n-1}}{1-\eta^{n-1}} & \int_{0}^{\eta} \frac{(\eta-s)^{n-1}}{(n-1) !} g_{p}(s) d s \\
& -\frac{t^{n-1}}{1-\eta^{n-1}} \int_{0}^{1} \frac{(1-s)^{n-1}}{(n-1) !} g_{p}(s) d s,
\end{aligned}
$$

where $g_{p} \in S_{F}\left(y_{p}\right)$.

Now, we consider the linear continuous operator $\Gamma: L^{1}([0,1], \mathbb{R}) \rightarrow C([0,1], \mathbb{R})$ defined by

$$
\begin{aligned}
\Gamma(g)(t)=\int_{0}^{t} \frac{(t-s)^{n-1}}{(n-1) !} g(s) d s+\frac{t^{n-1}}{1-\eta^{n-1}} & \int_{0}^{\eta} \frac{(\eta-s)^{n-1}}{(n-1) !} g(s) d s \\
& -\frac{t^{n-1}}{1-\eta^{n-1}} \int_{0}^{1} \frac{(1-s)^{n-1}}{(n-1) !} g(s) d s .
\end{aligned}
$$

We have $h_{p} \in \Gamma \circ S_{F}\left(y_{p}\right)$. From Lemma 2, $\Gamma \circ S_{F}$ has a closed graph, then $h \in$ $\Gamma \circ S_{F}(y)$. Thus, there exists a $g \in S_{F}(y)$ such that

$$
h(t)=\Gamma(g)(t), \quad t \in[0,1],
$$

which implies that $h \in T(y)$. Consequently, $T$ is upper semi-continuous.

STEP 5: The following set is bounded:

$$
\Omega=\{y \in C([0,1], \mathbb{R}): \lambda y \in T(y) \text { for some } \lambda>1\} .
$$

Indeed, let $y \in \Omega$. Then

$$
\begin{aligned}
y(t)=\lambda^{-1} \int_{0}^{t} \frac{(t-s)^{n-1}}{(n-1) !} g(s) d s+\frac{\lambda^{-1} t^{n-1}}{1-\eta^{n-1}} & \int_{0}^{\eta} \frac{(\eta-s)^{n-1}}{(n-1) !} g(s) d s \\
& -\frac{\lambda^{-1} t^{n-1}}{1-\eta^{n-1}} \int_{0}^{1} \frac{(1-s)^{n-1}}{(n-1) !} g(s) d s .
\end{aligned}
$$

where $g \in S_{F}(y)$. So, we conclude that 


$$
\begin{aligned}
\|y\|_{\infty} \leq \lambda^{-1} \int_{0}^{1} \frac{(1-s)^{n-1}}{(n-1) !} m(s) d s+\frac{\lambda^{-1}}{1-\eta^{n-1}} \int_{0}^{\eta} \frac{(\eta-s)^{n-1}}{(n-1) !} m(s) d s \\
+\frac{\lambda^{-1}}{1-\eta^{n-1}} \int_{0}^{1} \frac{(1-s)^{n-1}}{(n-1) !} m(s) d s .
\end{aligned}
$$

This shows that $\Omega$ is bounded. Thus, $T$ satisfies all the conditions of Lemma 3 . Therefore, $T$ has a fixed point which is a solution of (1.1).

Proof of Theorem 2. Transform the problem into a fixed point problem. Set for all $t \in[0,1]$

$$
\psi_{n}^{g}(t)=\int_{0}^{t} \frac{(t-s)^{n-1}}{(n-1) !} g(s) d s+\sum_{k=0}^{n-2} \varphi_{n}^{(k)}(t) \int_{0}^{\eta} \frac{(\eta-s)^{k}}{k !} g(s) d s,
$$

where $g \in S_{F}(y)$. Consider the multi-valued map $T: C([0,1], \mathbb{R}) \rightarrow 2^{C([0,1], \mathbb{R})}$ defined as follows: for $y \in C([0,1], \mathbb{R})$, one puts

$$
T(y):=\left\{z \in C([0,1], \mathbb{R}): z(t)=\psi_{n}^{g}(t)+\frac{1+t}{1-\tau}\left(\psi_{n}^{g}(\tau)-\psi_{n}^{g}(1)\right) \text { for } t \in[0,1]\right\} .
$$

Following the steps of the proof of Theorem 1, we can easily show that $T$ has a fixed point $y$.

Now we shall show that $y$ is a solution of (1.2) and (1.3). We have

$$
y(t)=\psi_{n}^{g}(t)+\frac{1+t}{1-\tau}\left(\psi_{n}^{g}(\tau)-\psi_{n}^{g}(1)\right),
$$

where $g \in S_{F}(y)$. Then $y(1)=y(\tau)$. On the other hand, for $0 \leq i \leq n-2$ and $t \in[0,1]$, we have

$$
\begin{aligned}
{\left[\psi_{n}^{g}\right]^{(i)}(t) } & =\int_{0}^{t} \frac{(t-s)^{n-i-1}}{(n-i-1) !} g(s) d s+\sum_{k=0}^{n-2} \varphi_{n}^{(k+i)}(t) \int_{0}^{\eta} \frac{(\eta-s)^{k}}{k !} g(s) d s \\
& =\int_{0}^{t} \frac{(t-s)^{n-i-1}}{(n-i-1) !} g(s) d s+\sum_{l=i}^{n+i-2} \varphi_{n}^{(l)}(t) \int_{0}^{\eta} \frac{(\eta-s)^{l-i}}{(l-i) !} g(s) d s \\
& =\int_{0}^{t} \frac{(t-s)^{n-i-1}}{(n-i-1) !} g(s) d s+\sum_{l=i}^{n-2} \varphi_{n}^{(l)}(t) \int_{0}^{\eta} \frac{(\eta-s)^{l-i}}{(l-i) !} g(s) d s .
\end{aligned}
$$

Then, by Remark 1(a) and (b),

$$
\begin{aligned}
{\left[\psi_{n}^{g}\right]^{(i)}(0) } & =\int_{0}^{\eta} \frac{(\eta-s)^{n-i-2}}{(n-i-2) !} g(s) d s+\sum_{l=i}^{n-3} \varphi_{n}^{(l)}(0) \int_{0}^{\eta} \frac{(\eta-s)^{l-i}}{(l-i) !} g(s) d s \\
& =\int_{0}^{\eta} \frac{(\eta-s)^{n-i-2}}{(n-i-2) !} g(s) d s+\sum_{l=i}^{n-3} \varphi_{n-l-1}(\eta) \int_{0}^{\eta} \frac{(\eta-s)^{l-i}}{(l-i) !} g(s) d s,
\end{aligned}
$$


and, by Remark 1(a),

$$
\begin{aligned}
{\left[\psi_{n}^{g}\right]^{(i+1)}(\eta) } & =\int_{0}^{\eta} \frac{(\eta-s)^{n-i-2}}{(n-i-2) !} g(s) d s+\sum_{l=i}^{n-3} \varphi_{n}^{(l+1)}(\eta) \int_{0}^{\eta} \frac{(\eta-s)^{l-i}}{(l-i) !} g(s) d s \\
& =\int_{0}^{\eta} \frac{(\eta-s)^{n-i-2}}{(n-i-2) !} g(s) d s+\sum_{l=i}^{n-3} \varphi_{n-l-1}(\eta) \int_{0}^{\eta} \frac{(\eta-s)^{l-i}}{(l-i) !} g(s) d s
\end{aligned}
$$

Consequently,

$$
\left[\psi_{n}^{g}\right]^{(i+1)}(\eta)=\left[\psi_{n}^{g}\right]^{(i)}(0),
$$

which implies that $y(0)=y^{\prime}(\eta)$ and

$$
y^{(i)}(0)=y^{(i+1)}(\eta), \quad 2 \leq i \leq n-2,
$$

whenever $n \geq 4$. Finally, it is clear that

$$
y^{(n)}(t)=g(t), \quad t \in[0,1],
$$

and, hence,

$$
y^{(n)}(t) \in F(t, y(t)), \quad t \in[0,1],
$$

as required.

Proof of Theorem 3. Consider the multi-valued map $T: C([0,1], \mathbb{R}) \rightarrow 2^{C([0,1], \mathbb{R})}$ defined as follows: for $y \in C([0,1], \mathbb{R})$, one sets

$$
T(y):=\left\{z \in C([0,1], \mathbb{R}): z(t)=\psi_{n}^{g}(t) \text { for } t \in[0,1]\right\} .
$$

Following the steps of the proof of Theorem 1, we show that $T$ has a fixed point $y$. Let us show that $y$ is a solution of (1.4). Indeed, by (4.1), we have (4.2). In view of (4.3), it follows that (4.4) also holds.

\section{ACKNOWLEDGEMENT}

The authors would like to thank the anonymous referee for his careful reading of the paper and for his pertinent suggestions.

\section{REFERENCES}

[1] M. Benchohra and S. K. Ntouyas, "A note on a three point boundary value problem for second order differential inclusions," Math. Notes (Miskolc), vol. 2, no. 1, pp. 39-47, 2001.

[2] M. Benchora and S. K. Ntouyas, "Multi point boundary value problems for second order differential inclusions," Mat. Vesnik, vol. 53, no. 1-2, pp. 51-58, 2001.

[3] C. Castaing and M. Valadier, Convex analysis and measurable multifunctions, ser. Lecture Notes in Mathematics, Vol. 580. Berlin: Springer-Verlag, 1977.

[4] A. Lasota and Z. Opial, "An application of the Kakutani - Ky Fan theorem in the theory of ordinary differential equations," Bull. Acad. Polon. Sci. Sér. Sci. Math. Astronom. Phys., vol. 13, pp. 781$786,1965$. 
[5] M. Martelli, "A Rothe's type theorem for non-compact acyclic-valued maps," Boll. Un. Mat. Ital. (4), vol. 11, no. 3, suppl., pp. 70-76, 1975, collection of articles dedicated to Giovanni Sansone on the occasion of his eighty-fifth birthday.

Authors' addresses

M. Aitalioubrahim

University Hassan II - Mohammedia, U. F. R. Mathematics and Applications, F. S. T., BP 146, Mohammedia, Morocco

E-mail address: aitalifr@yahoo.fr

S. Sajid

University Hassan II - Mohammedia, U. F. R. Mathematics and Applications, F. S. T., BP 146, Mohammedia, Morocco

E-mail address: saidsajidehotmail.com 